PROF-1338

\title{
DIABETIC FOOT INFECTIONS;
} ROLE OF GRANULOCYTE COLONY STIMULATING FACTOR(G-CSF).

\author{
DR. MUHAMMAD SAEED AKHTAR, FCPS \\ Assistant Professor of Medicine \\ Independent Medical College Faisalabad.

\section{DR. MAQSOOD AHMAD, FCPS} \\ Senior Registrar Medicine \\ Allied Hospital (PMC) Faisalabad.
}

\author{
Dr. Muhammad Irfan, MBBS \\ Resident Medical Officer \\ Allied Hospital (PMC) Faisalabad. \\ Dr. Zahid Yasin Hashmie, FCPS \\ Professor of Medicine \\ Punjab Medical College Faisalabad.
}

\section{DR. MUHAMMAD BADAR BASHIR, FCPS \\ Assistant Professor of Medicine \\ Independent Medical College Faisalabad.}

\begin{abstract}
Objective: (1) To evaluate the effects of G-CSF in eliminating infection in diabetic foot wound (2) To compare the effects with conventional diabetic foot management. Design: Prospective, open, randomized comparative study. Setting: Medical\&Surgical Department of Allied, DHQ Hospital \& Nawaz Medicare Faisalabad. Period: From Jan 2000 to Nov 2000 Patients \& Methods: Fifty diabetic patients with foot infections were included in this study. The mean age was 52 years ranging from 27 to 60 years. They were divided into two equal groups(Group A and Group B). Results: The male patients were $41(82 \%)$ and female $9(18 \%)$. Forty six percent of patients were on oral hypoglycaemic drugs, and $54 \%$ on insulin. The trauma preceding infection was $20 \%$, Peripheral neuropathy $94 \%$ and peripheral vascular disease $34 \%$. Thirty two percent of patients were smoker. Group A were subjected to G-CSF ( Neupogen ) therapy $(n=25)$ subcutanously daily for 5 days in addition to conventional measures. Whereas patients in Group $B$ received only conventional therapy. Both groups received similar antibiotic and insulin treatment. G-CSF therapy was associated with earlier eradication of pathogens from the infected ulcer (median 5 [range 2-11] vs11 [6-31] days in the group $B ;(p=<0.000 I)$, quicker resolution of cellulitis ( 6 vs 14 days; $p<0.0001)$, shorter hospital stay ( 8 vsl 6 days; $p<0.0001)$, and a shorter duration of intravenous antibiotic treatment (7 vs 14 days; $p-0.0001)$.No G-CSF treated patient needed surgery, whereas three patients in group B underwent toe amputation and six had extensive debridement under anaesthesia ( $p=0.00$ 1). G-CSF therapy was generally well tolerated. Conclusion: Granulocyte colony stimulating factor (G-CSF) may be used as a good adjuvant therapy along with conventional measures for the management of diabetic foot infection, as it promotes the healing of diabetic foot ulcer/cellulitis and consequently prevents many hazardous complications like amputation of limbs, long hospital stays, extensive and prolonged antibiotic use and last but not the least the total misery of the patients.
\end{abstract}

Key words: G-CSF, D. M. 


\section{INTRODUCTION}

The pedal manifestations of diabetes are well documented and potentially limb-threatening when left untreated. The evaluation of the diabetic foot involves careful assimilation of the patient's history and physical findings with the results of necessary diagnostic procedures $^{1}$.

Diabetes mellitus is a syndrome with disordered metabolism and inappropriate hyperglycemia due to either a deficiency of insulin secretion or to a combination of insulin resistance and inadequate secretion to compensate for ${ }^{2}$.

Diabetes mellitus is one of the commonest clinical endocrine disorders which is responsible for very high morbidity and mortality. Three hundred million people are expected to have the disease by $2025(5.4 \%)$, of which $170 \%$ increase is expected in developing countries. Urban dwellers are more likely to have the disease than rural inhabitants ${ }^{3,4}$.

Diabetes mellitus spares no organ or system of the body and produces both acute and chronic complications including coronary artery disease, peripheral vascular disease, cerebrovascular accidents, retinopathy and diabetic nephropathy leading to chronic renal failure, autonomic and peripheral neuropathy, and amputation of extremities.

An association between diabetes and foot problems had been recognized since long. In 1887, Pryce, an English surgeon, described a diabetic patient who developed peripheral neuropathy and later, ulcer on the plantar surfaces of his feet. He concluded, "Diabetes may of itself be a cause of perforating ulcers" .

Macroangiopathy takes a heavy toll among diabetics, being responsible for coronary heart disease, peripheral vascular disease and cerebrovascular accidents. Their relative risk of lower-limb vascular disease is 4.5 (Framingham study), while risk of lower-limb gangrene is 20-25 times higher than in general population. Half of all lower- limb amputations are performed in diabetics. Diabetic foot infection is a major health problem, accounting for a longer hospital stay than all other complication of diabetes It is not infrequent that treatment for one single foot problem exceeds the total annual cost for metabolic control ${ }^{6,7}$.

Diabetic foot problems occur both in insulin-dependent \& non-insulin dependant diabetes mellitus. These are more common in men and in patients over 60 years of age. $A$ recent population based study in over 10,000 patients in the North West of England reported past or present foot ulceration and almost $67 \%$ had one or more risk factors for diabetic foot. Foot ulcers are common in Caucasians than in Asians and Afro-Caribbean diabetic patients. Examination of the economic costs of diabetic complications reveals that the diabetic foot problems are responsible for $20 \%$ of all diabetic admissions to the hospital in both the United Kingdom and United States. The mean duration of hospitalization for amputation in one study was 27 days ${ }^{8,9}$.

\section{OBJECTIVES}

The aims of the study are

To evaluate the effects of Granulocyte colony stimulating factor (G-CSF) in eliminating infection in diabetic foot wound compared with conventional treatment in two groups under study.

\section{Primary Objectives}

Primary study objectives were time to resolution of infection (cellulitis), intravenous antibiotic requirements, and time to hospital discharge.

\section{Secondary objective}

Secondary objective were the need for surgery, and the time taken for pathogens to be

eliminated from the wound

\section{MATERIALS AND METHODS}

A total of 50 patients fulfilling the criteria mentioned below were studied for a period of 11 months between January, 2000 and November, 2000 who were admitted from Out Patient Department and in Medical/Surgical departments at Allied Hospital, Divisional Head Quarter Hospital, And Nawaz Medicare, Faisalabad. All participants were older than 18 years and gave informed 
consent.

Patients in Group A received G-CSF (Neupogen). G-CSF was administered as a daily subcutaneous injection for 5 days. The initial dose of G-CSF was 5 micrograms $/ \mathrm{kg}$ daily. The dose was lowered to 2.5 micrograms $/ \mathrm{kg}$ daily, if after two doses, the absolute neutrophil count was higher than $25 \times 10^{9} / \mathrm{L}$. If the absolute neutrophil count remained above this value after a further two doses, GCSF was given as 2.5 micrograms $/ \mathrm{kg}$ daily on alternate days. If at any point the absolute neutrophil count was greater than $50 \times 10^{9} / \mathrm{L}$ or the total white-cell count was greater than $75 \times 10^{9} / \mathrm{L}$ G-CSF was stopped until the absolute neutrophil count fell below $10 \times 10^{9} / \mathrm{L}$. Laboratory parameters were studied by the staff not involved in clinical assessment of the patients. Changes in the doses of G-CSF were done according to the values of TLC and DLC.

A combination of four antibiotics (ampicillin, cloxacillisn, ciprofloxacin and metronidazole) was given intravenously until cellulitis and ulcer discharge had resolved. If an infecting pathogen was identified before admission and enrolment, the appropriate antibiotics were used as firstline therapy. Subsequent changes in antibiotic ragmen were guided by microbial cultures and antimicrobial susceptibilities.

Glycaemic control was optimized with insulin in all patients, by means of a multiple-dose regimen.

Daily aseptic dressing of the wound was done.

\section{INCLUSION CRITERIA}

Diabetic patients with foot infections having age between 18-70 years who were interviewed using a standard questionnaire.

Patients with cellulitis (cellulitis defined as an acute spreading infection of the skin with involvement of the subcutaneous tissues, clinically characterized by erythema more than $2 \mathrm{~cm}$ in diameter) in association with purulent discharge, with or without lymphangitis.

1. Superficial ulcer
2. Deep ulcer

3. Deep ulcer with abscess formation with or without bony involvement

\section{EXCLUSION CRITERIA}

1. Absolute neutrophil count on presentation of less than $1 \times 10^{9} / \mathrm{L}$ or greater than $50 \times 10^{9} / \mathrm{L}$

2. History of previous or current malignant disorder

3. Serum creatinine more than $\mathbf{2 5 0}$ micromole per litre

4. Patients with abnormal liver function tests

5. Previous organ transplantation

6. Immunosuppressive therapy including corticosteroids

7. Pregnancy

8. Lactation

9. Multiple organ failure secondary to septicaemia

\section{STUDY ASSESSMENT}

Each day, patients were reviewed independently by two clinicians and the affected limb was assessed. Requirements for discharge from the hospital were that the cellulitis had resolved and there was no further exudate from the ulcer; and the cultures from the foot ulcer no longer yielded a pathogen. Any decisions about surgical debridement or amputation were based on clinical signs, including the presence of non-viable tissue, the development of gangrene, abscess formation, lack of improvement despite optimum antimicrobial therapy. All clinical decisions were made independent of study treatment and white-cell count. If a patient had several ulcers, the most severely affected ulcer was studied. Capillary glucose measurements were done four times daily with the help of Glucometer (Lifescan, One touch, Thonson \& Thonson company, USA.), before meals and at 2200 hours throughout the hospital stay.

\section{IDENTIFICATION OF RISK FACTORS}

Peripheral Neuropathy was considered to be present if there was a history of numbness in the feet, absence of pain or altered fine touch sensation and proprioception.

Peripheral vascular disease was defined as the presence of ischemic symptoms such as intermittent claudication or rest pain, and! or an absence of pedal pulses. 


\section{LABORATORY ASSAYS AND RADIOGRAPHY}

Standard haematological measurements were made by routine methods. Swabs from the deepest part of the wound were collected daily after ulcer cleansing with sterile saline and removal of superficial debris; swabs were shifted to the microbiology laboratory immediately in sterile container for aerobic and anaerobic cultures. Sampling was done until cultures were negative on two consecutive days.

\section{STATISTICAL ANALYSIS}

50 patients were divided into two groups of 25 each at random called group A \& B. Group A was treated with GCSF and conventional measures while group $B$ received only conventional therapy. To compare the effects of G-
CSF with respect to each trait, Mann-Whitney test was applied, because normal probability plots indicated nonnormality in the data (Analysis of normal probability plot suggested to use Mann-Whitney test and not to use ttest). BMDP computer package was used for statistical analysis. The differences were taken to be significant at $p<0.05$.

\section{RESULTS}

A total of 50 patients were included. Age ranged from 27 to 69 years. The major bulk of patients comprised of the group between $46-65$ years of age $(68 \%)$. Amongst this study sample $41(82 \%)$ were male and $9(18 \%)$ were female (table I).

\begin{tabular}{|l|c|c|c|c|c|c|}
\hline \multicolumn{7}{|c|}{ Table-I. Patient age groups and gender. } \\
\hline Age range & \multicolumn{2}{|c|}{ Group 1 } & \multicolumn{2}{c|}{ Group 2 } & \multicolumn{2}{c|}{ Total } \\
\hline & Male & Female & Male & Female & Male & Female \\
\hline $26-35$ & 3 & 1 & 2 & 1 & 5 & 2 \\
\hline $6-45$ & 1 & - & 3 & 1 & 4 & 1 \\
\hline $46-55$ & 9 & 2 & 7 & 2 & 16 & 4 \\
\hline $56-65$ & 6 & 1 & 6 & 1 & 12 & 2 \\
\hline $66-75$ & 2 & - & 2 & - & 4 & - \\
\hline Total & 21 & 4 & 20 & 5 & 41 & 9 \\
\hline
\end{tabular}

Mostly patients were recruited from diabetic clinic and medical/surgical units of Allied Hospital Faisalabad (80\%). Remaining 20\% came from medical/surgical units of Divisional Headquarter Hospital Faisalabad, and Nawaz Medicare Faisaiabad.

Body mass index of most of the patients ranged between $26-35 \mathrm{~kg} / \mathrm{rn}$. Forty three $(86 \%)$ patients had Glycated haernoglobin (HbAic) values between 7.1- 11.0. Thirty eight patients were of type2 diabetes while others were type 1 diabetics. Forty six percent of patients were on oral hypoglycemic drugs, and $54 \%$ on insulin.

Of the 50 patients, 25 were assigned to G-CSF in addition to conventional measures and were placed in
Group A and 25 patients received only conventional therapy and were put in Group B. There were no significant differences between the groups in clinical or demographic characteristics on entry to the study. The sites of the infected lesions were similar in the two groups. In the Group A, eighteen patients had infected ulcer in the forefoot, four had infected ulcer in the mid foot and three had infected ulcer in the hind foot. The corresponding numbers in the group $B$ were 20 , three and two. Eight patients in Group $A$ and seven in Group B had multiple ulcers. The median duration of the foot ulcer before entry in the study was 18.0 days (range 6 to33) in Group A and 14.Odays (8to30) in the Group B.

Cellulitis had been present for a median of 8days (2to18) 
in Group A and 7 days (3 to20) in Group B. A history of trauma preceding infection was present in $20 \%$. Twenty seven (54\%) patients (14 Group A, 13 Group B) had evidence of osteomyelitis, 15 patients with radiographic changes (eight Group A, seven Group B) and twelve with clinical signs (5 Group A, 7 Group B).

Retinopathy was present in $31(62 \%)$ patients, and peripheral neuropathy in $47(94 \%)$. Peripheral vascular disease was noted in $34 \%$ and $16(32 \%)$ had ischemic heart disease. Seven (14\%) patients had a history of cerebrovascular disease, and 21(42\%) had hypertension. A history of smoking was present in $16(32 \%)$, and nephropathy was detected in $21(42 \%)$. A previous history of amputation/debridement was present in 15(30\%) patients. There were no significant differences between the groups in these associated findings.(table II)

\begin{tabular}{|l|c|c|c|c|}
\hline \multicolumn{5}{|c|}{ Table-II. Diabetic complications and associated risk factors. } \\
\hline Associated finding & Group I & \%age & Group II & \%age \\
\hline Retinopathy & 16 & 64 & 15 & 60 \\
\hline Nephropathy & 11 & 44 & 10 & 40 \\
\hline Smokers & 9 & 36 & 7 & 28 \\
\hline History of IHD & 7 & 28 & 9 & 36 \\
\hline History of CVA & 3 & 12 & 4 & 16 \\
\hline History of amputation/debridement & 6 & 24 & 9 & 36 \\
\hline Hypertension & 10 & 40 & 11 & 44 \\
\hline Neuropathy & 23 & 92 & 24 & 96 \\
\hline Peripheral vascular disease & 10 & 40 & 7 & 28 \\
\hline
\end{tabular}

Pathogens recovered from wound cultures of $41(82 \%)$ individuals at baseline. Involvement of pathogens in two groups was almost comparable. Staphylococcus aureus was the commonest type of pathogen isolated. Gramnegative aerobic bacilli were slightly more common in Group B. However no individual species of gramnegative bacilli was commoner in either group. (Table III)

Patients who received G-CSF therapy had significantly earlier eradication of pathogens from wound swabs, quicker resolution of cellulitis, shorter hospital stays, and shorter duratio9n of intravenous antibiotics treatment than control group. No G-CSF treated patient needed surgery, whereas in the group $B$ three patients underwent toe amputation, and six had extensive debridement under anaesthesia on more than one occasions; this difference was also significant $(p=0.001)$.
Ten (40\%) patients had ulcers healed at day 7 in the GCSF treated group (Group A) compared with none in the Group $B(p=0.0005)$. In patients with multiple ulcerations, there was no deterioration in any secondary ulcers. Metabolic control did not differ significantly between the groups.

All patients received a multiple-dose regimen of human short-acting insulin given before meals.

The median G-CSF dose over 5 days was 300 micrograms/day (180-- 410); ten patients required a reduction in dose. Twenty three patients in Group $A$ and

21 patients in Group B received the antibiotic regimen ciprofloxacin (400mg daily), ampicillin (1.5 g daily), cloxacillin (1.5 g daily), and metronidazole ( $1.5 \mathrm{~g}$ daily); all antibiotics were given intravenously and then by 
mouth if appropriate.

\begin{tabular}{|c|c|c|}
\hline \multicolumn{3}{|c|}{ Table-III. Pathogens isolated. } \\
\hline & \multicolumn{2}{|c|}{ No of isolates } \\
\hline & Group I & Group II \\
\hline Positive wound culture & 21 & 20 \\
\hline \multicolumn{3}{|l|}{ Gram Positive aerobe } \\
\hline Staphylococcus aureus & 14 & 11 \\
\hline Streptococcus aglactiac & 1 & 1 \\
\hline Enterococcus faecal is & - & 2 \\
\hline \multicolumn{3}{|l|}{ Gram Negative aerobes } \\
\hline Enterbacter spp & 2 & 2 \\
\hline Eschrochia Coli & 2 & 1 \\
\hline Klebsiella Spp & - & 1 \\
\hline Proteus Spp & 1 & 1 \\
\hline Psuedomonas Spp & - & 2 \\
\hline \multicolumn{3}{|l|}{ Anaerobes } \\
\hline Bacteeriodes fragilis & 1 & 2 \\
\hline Others & 2 & 3 \\
\hline Polymicrobial & 7 & 9 \\
\hline
\end{tabular}

Patients with osteomyelitis received parenteral and/or oral therapy for at least 12 weeks. Initial treatment with ceftazidime ( $3 \mathrm{~g}$ daily) and gentamycin ( $240 \mathrm{mg}$ daily) was used in two patients with infection caused by pseudomonas species (Group B). Two patients m Group $A$ and 2 patients in Group B had standard therapy changed to imipenem/cilastatin for an infection with multidrug resistant Escherichia coil.

G-CSF therapy was associated with the development of leucocytosis, due almost entirely to an increase in neutrophil count. Total white-cell and neutrophil counts increased significantly after two doses of G-CSF, and the increases were maintained until day 7.There were also significant increases in lymphocyte and monocyte populations in patients receiving G-CSF. All the cell counts returned to near- baseline value within 48hours of the end of the therapy. There was mild change in lymphocyte or monocyte counts over7 days in Group B patients whereas neutrophil counts fell significantly. At day7, lymphocyte or monocyte counts were lower in patients of group B than in G-CSF-treated patients. (table IV).

Once treatment had started, no patient was withdrawn from the study. There were no protocol deviations, and no withdrawals of study medication because of side effects. A transient rise in serum alkaline phosphatase was observed in the group A (G CSF-treated group). Four patients in the group A (G-CSF-treated group) experienced transient bone pain that did not require analgesia.

\section{DISCUSSION}

Diabetic foot infections cause substantial morbidity and mortality. The cost for hospital care for diabetic foot problems is immense' Examination of the economic costs of diabetic complications reveals that the diabetic foot problems are responsible for $20 \%$ of all diabetic admissions to the hospitals in both the United Kingdom and United States. The mean duration of hospitalization for amputation in one study was 27 days The latter is a major financial burden to medical healthcare, as well as causing human suffering, prolonged functional disability, and associated mortality ${ }^{10,11}$.

Currie $\mathrm{CJ}$ et a1 conducted a study to compare the incidence and hospital costs associated with neuropathy and peripheral vascular disease (PVD) in patients with or without diabetes. This study also suggests a minimum prevalence figure for PVD, infection, neuropathy, and ulceration of $14.3 \%$ in the diabetic population In this study estimates suggest that patients with diabetes, who accounted for less than $2 \%$ of the population, consumed more than $20 \%$ of the resources allocated to this disease group $^{12}$.

Holzer SES et al conducted a study to investigate the costs of diabetic foot in the US, from the perspective of a primary healthcare insurer. He described that about 2$3 \%$ of patients with diabetes mellitus develop this 
complication each year, and $15 \%$ of patients with lifetime ${ }^{13}$. diabetes mellitus develop this chronic condition over their

\begin{tabular}{|c|c|c|c|}
\hline \multicolumn{4}{|c|}{ Table-IV. WBC counts during study } \\
\hline \multirow[b]{2}{*}{ Total white cell count } & \multicolumn{2}{|c|}{ Medium (range) cell count $\left(\mathrm{X}_{10} \mathrm{~g} / \mathrm{L}\right)$} & \multirow[t]{2}{*}{ P Value } \\
\hline & Group I & Group II & \\
\hline Base line & $7.2(4.9-14.20)$ & $7.1(4.6-12.2)$ & 0.4371 \\
\hline Day 3 & $20.0(12.5-31.0)$ & $7.1(4.6-12.2)$ & $<0.0001$ \\
\hline Day 5 & $26.0(20.3-42.0)$ & $7.0(4.5-12.1)$ & $<0.0001$ \\
\hline Day 7 & $24.0(14.8-46.50)$ & $6.9(4.4-12.0)$ & $<0.0001$ \\
\hline \multicolumn{4}{|l|}{ Neutrophils } \\
\hline Base line & $4.5(3.12-12.10)$ & $4.5(2.4-9.4)$ & 5.668 \\
\hline Day 3 & $17.4(10-27.7)$ & $4.5(2.3-9.2)$ & $<0.0001$ \\
\hline Day 5 & $22.3(17.5-38.6)$ & $4.5(2.3-9.1)$ & $<0.0001$ \\
\hline Day 7 & $22.1(12.6-43.7)$ & $4.5(2.1-8.9)$ & $<0.0001$ \\
\hline \multicolumn{4}{|l|}{ Lymphocytes } \\
\hline Base line & $4.5(3.12-12.10)$ & $4.5(2.4-9.4)$ & 5.668 \\
\hline Day 3 & $17.4(10-27.7)$ & $4.5(2.3-9.2)$ & $<0.0001$ \\
\hline Day 5 & $22.3(17.5-38.6)$ & $4.5(2.3-9.1)$ & $<0.0001$ \\
\hline Day 7 & $22.1(12.6-43.7)$ & $4.5(2.1-8.9)$ & $<0.0001$ \\
\hline \multicolumn{4}{|l|}{ Monocytes } \\
\hline Base line & $0.15(0.0-0.6)$ & $0.15(0.0-0.3)$ & 0.2684 \\
\hline Day 3 & $0.25(0.0-0.5)$ & $0.18(0.1-0.4)$ & 0.0054 \\
\hline Day 5 & $0.28(0.0-1.2)$ & $0.19(0.1-0.4)$ & 0.0046 \\
\hline Day 7 & $0.44(0.0-1.2)$ & $0.19(0.1-0.3)$ & 0.009 \\
\hline
\end{tabular}

Qari FA et al conducted a study to determine the mode of presentation and treatment of diabetic foot problems amongst 34 diabetic patients. Most patients were male, and mean age was 59 years. Sixty- five percent of patients were on oral hypoglycemic drugs, and $29 \%$ on insulin. A history of trauma preceding the infection was present in 20\%. Peripheral neuropathy was a factor in 94\%, and PVD in 50\%. Forty-four percent of patients smoked. Foot ulcer was the commonest type of presentation in $59 \%$ of patients, and eight ( $23 \%$ ) patients had a major amputation. The mean hospital stay was 21(3-39) days. Proteus and Pseudomonas were commonest, but MRSA (methicillin-resistant Staphylococcus aureus) was isolated in three cases. Most received a course of antibiotics (to cover both aerobic and anaerobic infections) for 3- 4weeks. Peripheral neuropathy as well as poor glycaemic control was common participating factors. Most patients needed 
debridement $22(65 \%)$, toe amputation in $11(23 \%)$ patients and eight (23\%) patients had a major limb amputation. A prolonged hospital stay was noted in the study ( $21 \pm 18$ days), and this is a common problem in the management of diabetic foot problems ${ }^{14}$.

The above-mentioned study dealt with subjects in a conventional manner. Conventional therapies were brought into vogue and even broad-spectrum antibiotics were used for a relatively extended period of time. In comparison with the above-mentioned study, in our study G-CSF was used in addition to conventional therapy, antibiotics were used for a shorter span of time. The results portrayed a totally different picture in one special aspect that is the number of amputations/debridement that became necessary in the study done by Qari FA et al' was 22 while in our study the same figure stood at nil. It is interesting to note that in our control group (Group B) the subjects showed almost the same results as those in above mentioned study.

Gough A et al' compared the efficacy of G-CSF in randomised placebo- controlled trial of granulocytecolony stimulating factor in diabetic foot infection. This study comprised of 40 patients who were randomly allocated to two groups. One group received G-CSF, while other group took placebo for 7 days. Both groups received similar antibiotic and insulin treatment. G-CSF therapy was associated with earlier eradication of pathogens from the infected ulcer (median4 vs. 8 days in the placebo group; $p=0.02$ ), quicker resolution of cellulites(7 vsl2 days; $p=0.03$ ), shorter hospital stay( 10 vs. 17.5 days; $p=0.02$ ), and a shorter duration of intravenous antibiotic treatment (8.5 vs 14.5 days $; p=0.02)$.No G-CSF treated patient needed surgery, whereas in placebo group two underwent toe amputation, and two had extensive debridement under anesthesia ${ }^{15}$.

Our study was to evaluate the effects of Granulocytecolony stimulating factor (G-CSF) when administered along with conventional measures in the management of diabetic foot infection, and to compare these effects with conventional therapy (Control Group). G-CSF therapy was associated with earlier eradication of pathogens from the infected ulcer, quicker resolution of cellulites, shorter hospital stay and a shorter duration of intravenous antibiotic treatment. No G-CSF treated patient needed surgery, whereas three patients in group B underwent toe amputation and six had extensive debridement under anaesthesia. These results are in line with above mentioned study.

The best predictor of lower limb amputation is a history of previous foot ulcer, the presence of neuropathy or peripheral vascular disease, and poor glycaemic control. Most of patients in my study had neuropathy and only $20 \%$ of patients were aware of trauma preceding foot infection

Peripheral vascular disease is another major predictive factor for diabetic foot ulceration' Smoking is an important risk factor for peripheral vascular disease $(32 \%$ of patients were cigarette smokers). Infections in diabetic patients are often polymicrobial in nature. There is a general agreement that diabetic patients have an increased rate of carriage of staphylococcus-aureus ${ }^{16}$. Swab cultures in my study were positive in $82 \%$, yielding a wide range of microorganisms. Staphylococcus aureus was commonest organism isolated in $64 \%$ of patients in my study.

Diabetic patients commonly fail to develop an appropriate neutrophil response to serious infection as reported by West $\mathrm{NJ}^{17}$. Our study also confirmed this finding. The administration of G-CSF in these patients resulted in rapid neutrophilic response. G-CSF therapy was well tolerated, and the side-effects seen were similar to those reported previously by Hollingshead LM' although high white-cell count may predispose patients to coronary and cerebral vascular events no acute vascular episodes occurred during G-CSF therapy in my study ${ }^{18,19}$.

It is to be mention that our study was the first of its kind conducted in Pakistan and similar kind of research has been done scantily the world over.

The final results exhibited a gross difference with the experimental group (Group A), who were given G-CSF in addition to conventional measures. The superior results seen in the Group A were clearly evident. Further the 
management of diabetic foot infection with the help of GCSF was found to be safe and effective. However, further multicentre studies involving a bigger number of patients are required to reach a more conclusive decision.

\section{CONCLUSION}

Granulocyte colony stimulating factor (G-CSF) may be used as a good adjuvant therapy along with conventional measures for the management of diabetic foot infection, as it promotes the healing of diabetic foot ulcer/cellulitis and consequently prevents many hazardous complications like amputation of limbs, long hospital stays, extensive and prolonged antibiotic use and lastbut not the least the total misery of the patients. G-CSF in the management of diabetic foot infection was shown to be safe and well tolerated. However large multicentre and comparative clinical trials should be conducted to confirm these findings.

\section{REFERENCES}

1. Frykberg RG, Zgonis T, Armstrong DG, Driver VR, Giurini JM, Kravitz SR, Landsman AS, Lavery LA, Moore C. Schuberth JM, Wukick DK, Andersen C, Vanore JV. Diabetic foot disorders: a clinical practice guideline. $J$ Foot Ankle Surg 2006 Sep-Oct;45(5):S2-66.

2. Johnll.Karam,MD: "D.M. and Hypoglycemia". In: Current Medical Diagnosis and Treatment. 39th ed., p: 1152,2000.Lange Medical Books/McGraw Hill.

3. UngerRR, Foster DW: "Diabetes mellitus" In: William's Textbook of Endocrinology.9 , 1997,W.B.Saunders company.

4. King, et at: "Diabetic Care",1998.

5. Pryce T.D.: "A case of perforating ulcers of both feet associated with Diabetes and ataxic symptoms". Lancet 2:11,1 887.

6. Fatani HH, Mira SA, El-Zubeir AG: "Prevalence of diabetes mellitus in rural Saudi Arabia". Diabetes Care 1987;10: 180-3.

7. DESG Teaching Letter: Number 6-Foot Care.

8. BoultenAJM; "The Diabetic Foot". In:Medicine 9, volume 11,1997.edition.
9. M.Benroubi,Greece; "Foot problems" In Medicographia,Volume 18, Supplement 1, 1996.p:34.

10. Marks HH: "Longevity and mortality of diabetics". Am J Public Health, 1965; 55: 416—423

11. Mcintyre BR, Deitch EA. "Diabetic foot infectionspathophysiology and treatment". Surg Clin North America 1994; 4: 537-55.

12. Young MJ, Boulton AJM, MacLeod AF, Williams DRR, Sonksen PH. "A multicentre study of the prevalence of diabetic peripheral neuropathy in the United Kingdom hospital clinic population". Diabetologia. 1993;36: 150154.

13. Holzer SES, Camerota A, Marten L, Cuerdon T, CrystalPeters J, et al. "Costs and duration of care for lower extremity ulcers in patients with diabetes". Clinical Therapeutics 20:169-181, Jan-Feb 1998. [American Academy of Otolaryngology-Head and Neck Surgery Foundation, Alexandria, Virginia, USA; Pharmaceutical Research Institute, Raritan, New Jersey, USA.

14. Muller MJ, Diamond JE, Sinacore DR. "Total contact costing in the treatment of diabetic plantar ulcers": controlled clinical trial. Diabetes Care 1989; 12:384-6.

15. Cebon J, Layton JE, Maher D, Morstyn G. "Endogenous haemopoietic growth factors in neutropenia and infection". Br Haematol 1994;86:265-74.

16. Casey $\mathrm{JL}$ "Host defense and infections in diabetes mellitus". In: Rifldn H, Prote D Jr. eds. Ellenberg and Rif kin's Diabetes Meffitus, Theory and Practice. 4 ed. Elsevier;1990:617-625.

17. West NJ. "Systemic antimicrobial treatment of foot infection in diabetic patients". Am I Health Syst Pharm 1995;52:1 199-1207.

18. Rollingshead LM, Gos KL. "Recombinant granulocytecolony stimulating factor(rG-CSF): a review of its pharmacological properties and prospective role in neutropethc conditions". Drugs $1991 ; 42: 300-30$.

19. Ernst E, Harnmerschmidt DE, Babbe $U$, Matrai $A$, DormandyJA. "Leukocytosis and the risk of ischaemic disease". JAMA 1987; 257:2316-24. 